\title{
Etnomarketing: estrategias de negocio desde las raíces culturales*
}

Nicolás Ortiz

Pontificia Universidad Católica del Perú

nortiz@pucp.pe

El presente artículo se enfoca en los cambios que se dieron en el estudio del consumidor a partir del enfoque colectivista que ofrece el etnomarketing en la era digital, el mismo que se deslinda de una visión racional e individualista. En primer lugar, se describen las principales dificultades que enfrenta el enfoque cerebral-racional, desde el cual se considera que el ser humano se diferencia de las demás especies por su capacidad de elegir libre y racionalmente. En segundo lugar, se propone un enfoque sociocultural de la antropología de consumo aplicada a los negocios -o etnomarketing- que evolucione hacia la visión del consumidor como alguien inmerso en diferentes contextos sociales y que organiza la compresión de su mundo a partir de su cultura. Finalmente, se realizan algunas reflexiones sobre los retos en la comprensión del consumidor en la era de las redes sociales.

Palabras clave: etnomarketing; antropología; consumo; marketing; comunicación

Etnomarketing: business strategies from cultural roots

This article focuses on the changes that took place in consumer studies starting with the collectivist focus that etnomarketing offers in the digital age, the same that separates itself from a rational and individualist vision. Firstly, the main difficulties that the cerebral-rational point of view confronts are described, point of view from which it is considered that human beings differentiate themselves from the other species by their capacity to choose freely and rationally. Secondly, a sociocultural approach of consumption anthropology applied to business (etnomarketing) that evolves towards the view of the consumers as immerse in different social contexts, and that organize their comprehension of the world starting from their culture, is proposed. Finally, some considerations are made about the challenges of understanding the consumers in the age of social networks.

Key Words: etnomarketing; anthropology; consumption; marketing; communication

\footnotetext{
* Este artículo está basado en la tesis de Maestría en Antropología por la Universidad Nacional Mayor de San Marcos Consumo de moda e identidad en jóvenes universitarios de Lima norte (2014) y en la ponencia "Consumo de moda e identidad en jóvenes emergentes: antropología del consumo" del Congreso de la Asociación Latinoamericana de Investigadores de la Comunicación (ALAIC 2014)
} 


\section{Introducción}

El principal dilema del mercadeo es anticipar los comportamientos de la demanda. Las motivaciones que orientan las elecciones de los consumidores no pueden entenderse como simples operaciones matemáticas frente al punto de venta o medio de comunicación, y es que el consumidor, más que un ente ponderador de elecciones, es un ser humano con reacciones instintivas, afectado por una mezcla de emociones e influenciado por un entorno social. Los motivos de compra del consumidor no pueden entenderse únicamente como decisiones racionales de estímulo-respuesta bajo el esquema del conductismo clásico, sin entender el contexto a través del cual interpreta sus necesidades, marcas, medios y productos elegidos. En este nuevo escenario de comprensión se encuentran hoy involucradas disciplinas como la biología, psicología y las ciencias sociales, cuyo afán no solo es analizar las motivaciones del consumidor, sino accionarlas efectivamente.

Para Deleuze, filósofo del conocimiento, la modernidad supone (por definición) esperar lo intempestivo frente a la repetición: cambios acelerados, globalización de la información, los mercados y los consumidores. Y los sujetos no se comprenden como personalidades estancas, sino en constante reinvención: "Somos lienzos ahumados de influencias pasadas, con rastros de otras vidas vividas (...) Somos desiertos, pero desiertos poblados de tribus, de faunas y de floras" (Deleuze 1980: 15).

El consumidor ha sido frecuentemente aislado en sus referentes personales y culturales, aglutinándosele de forma artificial y estadística. Los estudios del consumo deben entender la fuerza del contagio social y la influencia del entorno en los comportamientos; más allá de la obsesión métrica, la repetición del lenguaje oficial y la noción de laboratorio. Entender al consumidor con las categorías socio-económicas clásicas de compartimentos estancos, con baja carga empática y adaptación al contexto, requiere nuevos retos académico-empresariales.

\section{Paradigmas sobre el comportamiento del consumidor}

\subsection{Subjetividad del consumidor}

La construcción del ideal moderno de la racionalidad humana y sus decisiones individuales se torna una ideología en la era de la llustración, en la búsqueda por derrocar los dogmas místicos. Ello se debe a su aspiración de elevar la condición animal del hombre común para convertirlo en ser supremo, lo que justificaría su dominio sobre la naturaleza y sus recursos. El ser humano se diferenciaría de las demás especies por su libre capacidad de optar racionalmente, desafectando impulsos naturales, afectivos o de grupo para su homogenización y liberación. La guillotina sería el medio simbólico para derrocar el estado pre-racional de la especie humana, y confrontar al individuo separándolo del cuerpo social, en un afán por evolucionar hacia una sociedad "civilizada".

El racionalismo ha gobernado el estudio del comportamiento a nivel político, social, laboral e incluso de mercado. En algún momento de la historia, se aisló a la persona en una prisión, buscando ilusoriamente su control a partir de la métrica poblacional, con el afán de evitar a toda costa la disrupción. La misión fue otorgar a cada persona una casilla de la 
cual no podría moverse, sin contacto con los demás. Aislarnos desde el principio, asignarnos un número de identificación, agrupándonos en pabellones de indistintos ordenados por semejanzas, sin asumir la posibilidad de contagio por la era o la sociedad que nos rodea. El reconocimiento de nuestras creencias, sentimientos y manifestaciones se fue reemplazado por logaritmos. Primó la sensación de control al dividirnos, de contraponer personas y sus grupos de referencia, antes que la auténtica pasión por comprender que los sujetos funcionan la mayor parte del tiempo en colectividades.

En los estudios de la conducta del consumidor, la teoría economicista hereda los principios racionales del ser humano constriñendo a la toma de decisiones de consumo en las operaciones realizadas por el córtex o corteza cerebral, que opera la razón y la lógica empleada para entender la realidad consciente. Ello supone reducir las elecciones de consumo a una evaluación de alta implicación racional e individual entre las variables de calidad esperada sobre el precio.

Esta teoría, sobre la que se basaron extensamente los supuestos de la economía conductual para intentar predecir ciclos económicos o tendencias del consumo, se ve excedida o limitada en su infalibilidad empírica, lo que cuestiona su esencia científica. Ante ello, surgen nuevos enfoques que buscan ampliar los diferentes escenarios e influencias en las tomas de decisiones del consumidor para entender sus motivos y, más ambiciosamente aún, intentar desarrollar modelos predictivos.

Para entonces, en un escenario de crisis económica y escasez, la teoría neoclásica era considerada suficiente para entender el rol del sujeto como consumidor. Esta noción apela a un consumo racional del dinero en función de la calidad percibida y, ante la escasez de demanda y de una oferta variada y de suficiente calidad, las decisiones se realizaban desde la disponibilidad de los recursos y accesibilidad. Pero, incluso en estas circunstancias, los factores emotivos, subconscientes, culturales o tradicionales siguen operando de forma diversa, lo que hace inestable el modelo de predictibilidad de los ciclos económicos de la demanda. El consumidor se va consolidando como un ente complejo y decisivo en las dinámicas económicas, con capacidad de intervenir en sus ciclos, para lo cual se incorporan estudios que permitan medir las perspectivas de optimismo económico, percepción de bienestar o índices de calidad (que siempre es perceptual) de vida.

Entender que el ser humano no puede interpretar sus propios actos ni explicarlos es la base de las técnicas de comprensión de consumidores "predeciblemente irracionales" (Ariely 2008: 17). Estos métodos, que llamaremos posmodernos, de comprensión del consumidor, y con ello del marketing y la comunicación, se basan en la división del entendimiento (cerebro) en niveles más allá del pensamiento cognitivo.

\subsection{Retribalización del consumidor}

Con el desarrollo del internet, los medios masivos y el consumismo, las profecías más comunes vaticinaban el aislamiento del consumidor frente a un aparato electrónico. Por el contrario, el sociólogo Marshall Mcluhan, en los años sesenta, propuso el inicio de una aldea global donde los sujetos en el mundo se reconectaban a través de mensajes y medios compartidos, rompiendo barreras de espacio y tiempo. En pleno tercer milenio, en la era de las redes sociales, podemos entender que los sujetos y sociedades se retribalizan, lo que 
hace imposible seguir analizando al consumidor en solitario sin comprender su entorno, sociedad y cultura. Sin embargo, lo que estaríamos presenciando es que, en la era de las redes sociales, la generación de jóvenes contemporáneos rompe las barreras de espacio y tiempo, y nos enfrentaríamos a un proceso de retribalización de sujetos y colectivos en los universos virtuales, pero, a la par, esta generación se estaría desconectando de sus entornos sociales inmediatos. Ello nos estaría obligando a modificar los enfoques de investigación y comprensión de las identidades de personas y colectivos en las sociedades contemporáneas.

Los consumidores inmersos en la era de las redes sociales y el consumismo contemporáneo no se encontrarían encerrados en una celda egoísta ni desconectados de su entorno, sino en constante dinámica, multiplicando identidades a partir de sus motivaciones y actos de consumo. Esto permite dar pie a la formación de una escuela antropológica del consumo, entendiendo esto como un acto definitorio de los devenires personales, sociales y culturales del mundo contemporáneo.

Asimismo, en la era digital, se anuncia el fin del consumidor encerrado en una celda egoísta de desconexión con el entorno. Esta construcción imaginaria ha dado paso a un nuevo marketing, en el que se integre holísticamente al consumidor al comprender las claves sociales y culturales que movilizan sus ritos, mitos y formas de agrupación, lo que forja sujetos colectivos de consumo.

En términos del psicoanalista Julio Hevia, la personalidad se convertiría en un personal-deidad que explicaría todo y, a la vez por aislante y estereotípica, no explicaría nada del comportamiento concreto del consumidor (Hevia 2003: 81). Asimismo, el comportamiento del consumidor no puede ser entendido como una ciencia de sumatorias de individualidades, sin una adecuación a su entorno geográfico y social, tiempo histórico, lenguaje, creencias, formas de agrupación y percepciones.

La investigación psicológica del consumo, bajo la influencia del psicoanálisis, ha sabido especializarse en catalogar la comprensión de la sociedad urbana bajo el análisis de las pulsiones básicas y la satisfacción de las ansiedades, pero sus límites hasta hoy parecen extenderse desde las clases medias hasta las altas. La justificación de ello es que estos segmentos suponen ser el modelo y la referencia del resto de la población, sobre todo cuando estamos frente a ciudades con un centro de poder económico, social y simbólico unificado en la población pudiente. Este sector tiene el rol de normar los patrones que han de regir desde lo moral hasta lo estético, donde la moda es una herramienta de emulación deseable y piramidal en sus capacidades de acceso, lo que genera una regla tácita de aspiracionalidad no siempre verificada y que correspondería a modelos protourbanos ${ }^{1}$. Esta clase media tradicional, sin embargo, no estaría marcada por un espíritu inclusivo ni igualitario en nuestro país, lo que le resta su propia capacidad de influencia.

1. Dice Díaz-Albertini: "Al crecer nuestra sociedad en población, complejidad y heterogeneidad, ya no era posible marcar territorio de acuerdo al criterio de familia conocida. De ahí que se formaliza el proceso de selección y de acceso a los espacios considerados de gente como uno" (Díaz-Albertini 2000: 34). 


\section{Antropología del consumo: ¿una disciplina?}

La antropología, inevitablemente, implica un encuentro con el Otro. Con excesiva frecuencia, sin embargo, la distancia etnográfica que separa al lector y al antropólogo mismo del Otro se exagera. Este distanciamiento conduce a una focalización del otro como algo primitivo, curioso, exótico. La brecha entre el nosotros y el exótico ellos es un obstáculo fundamental para la comprensión significativa del Otro (Clifford Geertz 1989: 24).

En la era posmoderna el consumo se convierte en la actividad socializadora de los trabajadores, de la vida familiar y en la finalidad de la actividad laboral. La posmodernidad tiene como condición la universalidad de su conexión y medios, que se alimentaría de la diferencia para nutrirse en forma constante de sus contenidos. En ese sentido, el antropólogo posmoderno dejaría el paradigmático rol de defensor de permanencias para convertirse en un cronista de cambios e intercambios; posmodernidad que, en su multiplicidad de contenidos, es la fuente de materias de estudio en un proceso creciente de exploración de conocimientos colectivos y formas de socialización.

Las urbes cosmopolitas como París, Nueva York o Shanghái son hoy un crisol donde confluyen diferentes sociedades y se ejecutan intensos procesos de aculturación entre las comunidades locales, originales o extranjeras, lo que desplaza las fronteras de la alteridad desde la periferia hacia la urbe; esto es propio de su proceso de magnetismo sociocultural. Hablamos de fronteras en plural porque, más allá de la disputa entre lo extranjero y lo local, hoy en desuso en las grandes urbes, aparecen las confluencias migratorias, la movilidad social y las convergencias comerciales; ello sumado a la concentración corporativa de los negocios y de las marcas comerciales que van unificando los discursos de la oferta con sus promesas de bienestar, satisfacción, capacidad de elección, necesidades reales y percibidas, entre otros valores promovidos. Este es un reto que la antropología urbana acoge, marcada por las dinámicas que los mercados imponen en las interacciones sociales, lo que genera un magma de realidades sociales en movimiento ${ }^{2}$

La antropología cumple una labor clave en la vida urbana, en los espacios de retribalizaciones generados a partir de innumerables interacciones sociales propiciadas por el consumo; aldea global que integra la vastedad de mundos dispersos a través de las redes de telecomunicación, en un escenario de reintegración grupal y conexión intercomunitaria sin precedentes en la historia. En este escenario, la antropología entra a tallar en un rol amplio de descripción de nuevas agrupaciones en pleno proceso de intercambio y transformación, una labor cronista desde su capacidad interpretativa de los puntos de contacto, la intersección cultural, las transformaciones constantes como constante devenir. Al ser traída de vuelta a la ciudad, la importancia de los estudios de la cultura y los comportamientos neotribales en esta nueva aldea global 3.0 constituye la clave cultural que ayuda a describir y entender cómo piensa el colectivo, a descifrar lo que los une a los otros miembros, las convergencias, las convicciones compartidas. Más allá de una mirada polémica

2. Para Signorelli: "La antropología urbana se caracterizó como una antropología en la ciudad, es decir como una orientación de investigación que ponía en el centro de su interés la recuperación del contexto urbano de sus tradicionales objetos de investigación: familia y parentesco, grupos locales y vecindarios, tradiciones y rituales, todos objetos que permitían al antropólogo continuar utilizando los instrumentos conceptuales y metodológicos que la tradición de su disciplina le ofrecía" (Signorelli 1999: 70). 
al aprovechamiento comercial y del sistema capitalista de una disciplina, esta también es una oportunidad de comprensión de un nuevo ser humano y de las sociedades que se enfrentan a intensas dinámicas económicas pero con los mismos desafíos frente a la libertad de elección, asociación libre por intereses comunes, inclusión social ahora mediada por mercados cada vez más exigentes, democracia pero de consumidores frente a un poder influenciado por corporaciones internacionales; en suma, modelos de desarrollo que se vuelven permeables a diferentes ópticas particulares y culturales, hoy más visibles y activas que antaño.

\subsection{La inmersión antropológica en el consumo}

La antropología del consumo surgiría de la necesidad de entender la influencia de la cultura y sociedad en las decisiones de los sujetos contemporáneos que recurren al mercado para subsistir, competir y acopiar significados simbólicos para su vida cotidiana. Esta disciplina, al operar sobre la materia objetiva y subconsciente de la conducta, nutre de estudios culturales las formas en las que las mercancías adquieren valores simbólicos para las personas, más allá de sus beneficios instrumentales; simbolismo o consensos subjetivos sobre los que girarían las dinámicas de las economías y sociedades modernas. La sociedad estándar, anclada en la tradición que la psicometría ha buscado sistematizar, debe ser enriquecida con la experiencia en la contingencia que la antropología se ha especializado en tratar de comprender desde sus aportes a los estudios culturales.

Pero esto no es reciente. La antropología tiene origen comercial, siendo los antropólogos funcionarios de avanzada mercantil hacia otros mundos (América, Asia, África, Oceanía, Europa del Este) para traer el botín más valioso que asegure el éxito de los consorcios para-empresariales: el código cultural. Con este, los recursos naturales y las colectividades podían ser comprendidos y controlados.

Desde 1899, Thorstein Veblen, sociólogo interesado en cruzar los estudios de la economía con la antropología, parte de los estudios del mercado y el capital de Marx para analizar los primeros estudios de ciencia social del efecto comportamental del mercado: el comportamiento del consumidor. Su primera teoría sobre el particular, la emulación pecuniaria, analiza la evolución del consumo en las sociedades con una finalidad directa de ostentarla en sociedad, como forma de lenguaje para identificarse, comunicar la posición social y generar mecanismos de interacción entre grupos familiares como célula económica y social básica:

(La emulación pecuniaria es) afán de encumbramiento social, esa pasión por distinguirnos unos por encima de otros que, en todos los grupos sociales, da origen a las más singulares estrategias de diferenciación. Por ello cualquier gasto, por derrochador que pueda ser en realidad, debe tener, por lo menos, alguna excusa aceptable en forma de finalidad ostensible (Thorstein Veblen 1955: 51).

En 1988, Pierre Bourdieu, connotado sociólogo francés, presenta un análisis sociológico de las percepciones y consumos estéticos de la sociedad burguesa francesa a través de su consumo de bienes culturales y hábitos basado en un estudio cuanti-cualitativo 
de la sociedad francesa. Mary Douglas y Baron Isherwood, en 1990, publican El mundo de los bienes. Hacia una antropología del consumo buscando conciliar un análisis de las estructuras económicas de la sociedad contemporánea desde una perspectiva de análisis social y cultural. El consumo se apropia temporalmente de la dialéctica material-simbólico, integrando ambos ejes como parte de una misma dinámica en la cual los consumidores consumimos símbolos a través de la posesión de los bienes para dar sentido a nuestras vidas, como antes lo hacíamos a través de la religión o la política.

Douglas e Isherwood proponen, desde los estudios de antropología económica, una antropología del consumo que concilie el análisis social y cultural del intercambio de bienes (Douglas y Isherwood 1990: 79). Una mirada instrumental del consumo como un lenguaje o vehículo socializante; una suerte de puente entre el sujeto y los colectivos con los que se vincula. Los terrenos del consumo y la cultura son espacios dinámicos y confrontacionales: "el consumo es, por antonomasia, la arena en donde la cultura es motivo de disputas y remodelaciones" (Douglas y Isherwood 1990: 72).

Desde este enfoque, el consumo adquiere una relevancia de estudio social que lo aleja de la esfera superficial que las teorías sociales críticas le impusieron bajo la óptica del consumismo; se propone una ruta "hacia una antropología del consumo" que concilie el análisis social y cultural del intercambio de bienes (Douglas y Isherwood 1990: 18). Entonces, diríamos que los productos son símbolos más que objetos en la actualidad, y que tienen la capacidad de organizar el sentido de las vidas de aquellos que los consumen, tanto como la religión o la política lo hicieron en etapas previas.

Para Marc Augé, los nuevos espacios de consumo son también ambientes de transformación social e individual, dado que el consumo dinamizaría la cultura desde aquellos cambios íntimos que la globalización produce a nivel de las personas: "No hay identidad sin la presencia de los otros. No hay identidad sin alteridad" (Augé 1998: 57). El consumo se convierte parte de aquellos cambios íntimos que la globalización produce al nivel de las personas, a partir de espacios compartidos y objetos co-deseados. El punto de encuentro para el análisis es el shopping center, como nuevo universo de sentido "atomizado" de un universo integrado que es hoy el mundo. Los malls integran diversos sectores sociales y económicos, géneros y roles sociales. A través de estos espacios universales que se llaman "no lugares sin historia que afectan nuestras representaciones del espacio, nuestra relación con la realidad y nuestra relación con los otros" (Augé 1998: 83). Espacios desanclados con las realidades locales que crean contextos de socialización en una lucha entre el reconocimiento y la indiferenciación. Lejos de ser una disciplina encargada de la contención cultural, la antropología hoy tiene un escenario amplio y diverso para multiplicar las miradas sobre las nuevas formas socializantes. ${ }^{3}$

El consumo crea objetos de deseo compartidos, teniendo como punto de encuentro los shopping centers o malls, donde las individualidades se amalgaman en estilos de vida configurados por las fronteras de los espacios universales denominados "no lugares". Sin embargo, si bien este concepto es inmediatamente replicable en ciudades cosmopo-

3. "Y precisamente en este punto es donde se sitúa ahora el problema de la antropología. La razón consensual solo le dejaría a la antropología la tarea de hacer inventarios de patrimonios; la razón posmoderna le asignaría la tarea de orquestar sin otras pretensiones algunos ecos de la polifonía mundial" (Augé 1998: 55). 
litas, en nuestra realidad latinoamericana los espacios de consumo modernos conviven al lado de los canales tradicionales, por lo que se manifiesta una alternancia entre ambos lugares:

Aquí no se puede jugar con las categorías salvo que se manifiesten como contra-hipótesis, ya que debemos de tener en cuenta que los no lugares peruanos en términos históricos inter-relacionales e intersubjetivos (supermercados, metros, aeropuertos, hospitales, etcétera) son inexistentes ya que bien pueden presentarse con las lógicas identitarias nuestras y, desde luego, con sus características propias pero cuyos cauces difieren de los europeos o estadounidenses (Jacinto 2012: 68).

Y específicamente para los antropólogos, el estudio de los procesos de consumo podría ser una veta de oportunidad de nuevas dimensiones sociales más que una labor de análisis de contención cultural: "Mientras que para los economistas el consumo es en última instancia una destrucción, en los trabajos antropológicos se enfatiza más bien su aspecto creativo" (Huber 2002: 24).

En este proceso de reelaboración, el consumo juega un papel central, ya que es el motivador de las interacciones, así como el medio para las mismas, pues establece los puntos de contacto. El uso de medios de comunicación, comprendidos como un consumo cultural, es parte del proceso de influencia que promueve constantes escenarios de transformación. Es un cambio de identidad que las marcas mejor logradas ofrecen desde la experimentación de sensaciones, la simulación de ideales de conducta o simplemente la transformación de un estado potencial en una realidad deseada que el consumo viabilizaría; ofertas de transformación que, más que satisfactores, se convierten en el motor del encuentro entre la oferta y la demanda.

Asimismo, Hofstede define cultura como "programación mental colectiva de las personas en diferentes ambientes sociales". En su investigación sobre cultura y organizaciones (Hofstede 2010: 6), desarrolló un modelo, basado en cinco variables, que refleja las características y dimensiones culturales de cada país. Las empresas pueden ser capaces de manejar y comprender las diferencias culturales existentes y su influencia en los negocios.

Es en el subconsciente donde la etnología se encuentra con el psicoanálisis, y ambas buscan una comprensión del inconsciente colectivo, en este caso del consumidor. Estos drivers del consumo se codificarían en forma de normas que nos permitan determinar lo válido e inválido para la supervivencia física y social. Es adoctrinado por los padres y tutores, y reforzado por la experimentación y el grupo de pares. Estos valores, al tornarse tácitos e inconscientes, se asumen como verdades absolutos y operan sobre la realidad social en la forma de normas o reglas comunes no escritas.

La cultura es la forma de compresión del mundo en una sociedad específica. Opera como una lógica instalada previamente por el consenso social. Sus experiencias de socialización son el inicio del aprendizaje de los valores de su grupo y sociedad. Cuando aprenden que tienen valor para los demás, inicia su intercambio cultural. Y los bienes son parte de estas transacciones que se convierten en símbolos que tienen un valor personal y colectivo. Las claves sociales y culturales organizan el resto de sentidos para orientar la sobrevivencia y las vivencias sociales, las cuales son diferentes en cada cultura y contexto geográfico. 
La cultura se aprende cuando empieza el entrenamiento de los sujetos para enfrentarse a la sociedad. Y es que los seres humanos necesitan del contacto con otros para desarrollarse y satisfacer sus necesidades desde las más básicas hasta las plenas.

Es así como esta lógica del contagio social y del entorno se sofistica hacia el estudio de la cultura como madre del comportamiento del sujeto. Desde que educa en las palabras (y su uso adecuado en cada contexto), en diferenciar varones de mujeres, amigos de enemigos, lo bueno de lo malo, los valores asociados a los colores y sonidos la familia comienza a ser el primer agente oficial de lo que la sociedad a la que pertenece le instruye al niño-sujeto no solo para comprender la realidad, sino, lo más importante, cómo debe comportarse.

Influenciados por las escuelas norteamericana, inglesa y francesa, los recientes avances en marketing y estudios del comportamiento del consumidor proponen interpretaciones etnológicas a los estudios del consumidor: estudio de potencialidades, sistemas simbólicos, connotaciones religiosas, rituales, producción de mitos, etcétera. Sin embargo, la teoría social del consumo ha sido restringida al ámbito de la compulsión consumista, ya que se ha postulado una mirada crítica al consumo. De alguna manera, se forjó una valoración negativa de las estrategias de mercado y publicitarias, la economía especulativa, el imperio de la simbología de marcas y la oferta comercial de bienestar.

Debido a la importancia del análisis etnológico-cultural para asimilar productos y marcas a la simbología de los mercados, se han incorporado a la oferta académica y empresarial expertos o círculos de estudios culturales del consumo conocidos como las Teorías Culturales del Consumo (Consumer Culture Theory), que integran los estudios culturales, que son una ciencia interdisciplinaria liderada por la antropología junto con la literatura, arte, comunicación, entre otras.

Detectar los códigos culturales de los segmentos consumidores nos ayudará, entonces, a describir y entender cómo piensa el colectivo, a descifrar lo que los une a los otros miembros, las convergencias, las convicciones compartidas.

\subsection{Importancia de la antropología del consumo en el Perú de hoy}

En el Perú, ante el constante crecimiento de la capacidad de gasto de los sectores emergentes de Lima y de las ciudades de provincias, se producen también cambios en los estilos de vida y sus culturas originarias, generándose la reconfiguración de sus ejecuciones de gasto, lo que se une a los cambios en sus expectativas de vida, acceso informativo, íconos y modelos de conducta.

Los constantes cambios en el mercado son tanto producto de los sistemas políticos y económicos en conflicto que influyen en la producción y en la capacidad adquisitiva. En el Perú, el cambio de modelo económico estatista, desde el nacionalismo militar (1968) hasta el intervencionismo del primer gobierno aprista (1985-1990), dio paso a un nuevo modelo de apertura progresiva de mercados, liberalización de los servicios públicos y precios.

Paralelamente, la región sudamericana también se vio expuesta a cambios democráticos con el fin de dictaduras militares; la negación del modelo comunista que se transformó en un ciclo pendular entre el estatismo y mercantilismo; el acceso de nuevos sectores emergentes en las dinámicas del mercado; la globalización descentralizada junto con la 
proliferación del inglés y castellano, de la mano con la privatización abrupta de las empresas de telecomunicaciones a merced de capitales mexicanos o españoles; y cambios políticos, sociales y estructurales a nivel regional y nacional que tienen directa implicancia sobre las dinámicas en los puntos de venta, la percepción de necesidad y superficialidad, las perspectivas de futuro, la disposición al gasto y los valores simbólicos de los productos.

La mercadotecnia, a través de los estudios de Rolando Arellano y otras consultoras de investigación de mercado, ha visibilizado la población de Lima Norte a partir del auge económico y comercial de la zona. Catalogados como "emergentes", "progresistas", "emprendedores", sus comportamientos y perfil son constreñidos por la mercadotecnia a una visión económica y consumista, pero uniformizando sus variantes actitudinales, de gusto o culturales (Arellano 2010: 59).

La modernidad y globalización nos traen a diario información de un mundo más amplio y resistente. Pero también la realidad mediática nos habla de una nueva generación de jóvenes, hijos de migrantes, empoderados con el internet, los idiomas, educación superior, etc; es decir nuevos sujetos hechos protagonistas de la sociedad y el consumo cultural a los que el marketing tradicional no tenía contemplados por el ostracismo de las escuelas de negocio en las que se estudian solo negocios y se olvida la regla fundacional de la economía, la misma que dice que los ciclos económicos son los ciclos de la historia construida por los hombres en sus devenires intempestivos.

\section{Etnomarketing: estrategias desde las raíces culturales de la sociedad}

Se han generado ámbitos de aplicación empresarial y laboral, introduciendo sus modelos a los procesos empresariales de reclutamiento de personal, dirección de personas, evaluación y mercadeo. Los estudios del consumo, trabajando de la mano con la industria publicitaria, han logrado combinaciones para formular teorías y prácticas de mercado conjuntas, buscando medir sus actos en términos de efectividad de ventas.

El marketing y la publicidad se preguntan por aquellas desviaciones de lo que no encaja más en sus lógicas racionales de mercado; por esas tendencias que se van convirtiendo en masas de realidad. Ante el avance del acceso a la oferta y los mercados, descentramiento de las fuentes de irradiación socio-cultural, y multiplicación de los canales de venta, se impone una lógica social de comprensión de la conducta humana.

La confluencia de los estudios culturales del consumo con la gestión de negocios ha aportado nuevos enfoques a las ciencias empresariales por su carácter descriptivo, interpretativo y prospectivo de las tendencias sociales y de comportamiento.

Esta especialidad se ha denominado etnomarketing (Badot y otros 2009), mezcla de las labores etnográficas de registro social y cultural aplicada al conocimiento del consumidor y trabajador. Se busca entremezclar la mercadotecnia con la administración de la oferta y la demanda para controlar los flujos comerciales y los mensajes que orientan el consumo.

El etnomarketing surge de la necesidad de entender la influencia de la cultura, y sociedad, en las decisiones del consumo para activar estrategias. Se basa en la etnología (o estudio de las culturas) aplicada al estudio del consumidor o el comportamiento del comprador, con el objetivo de lograr elementos accionables. Su método de estudio, la etnografía, pretende revelar los significados que sustentan las acciones e interacciones 
que constituyen la realidad social del grupo estudiado; esto se consigue mediante la participación directa del investigador (Badot y otros 2009). Interesante también es el aporte de Dagoberto Páramo, que sistematiza la aplicación organizacional de la cultura "como el marco y la esencia del accionar de los negocios contemporáneos, tanto desde el consumidor como desde la organización y el mercado en el que ella se desenvuelve, y termina por poner a prueba, de manera decidida, el aporte hecho por la antropología, la etnografía y el simbolismo que estimula el fenómeno de consumo que caracteriza a las culturas de consumo actualmente reconocidas" (Páramo 2008: 11).

El etnomarketing supone un viaje al contexto de vida del consumidor, descolocarse para colocarse de nuevo (desnudarse para vestirse de nuevo). El etnomarketing no es un método. Es una inmersión y proceso de cambio de mentalidad del investigador. Un proceso filosófico de generación de conocimiento basado en la comprensión de una sociedad específica a partir de la religión, historia, economía, política, sociedad, arte, lingüística, esoterismo, etc.

El viajero, a diferencia del turista, se desplaza a un lugar diferente con maletas grandes y vacías; lo hace la disposición de involucrarse con el nuevo entorno y vivir un sinnúmero de experiencias interpersonales. El contacto con el público visitado es el medio para resolver las circunstancias. Traer experiencias, objetos y aprendizajes de vuelta. Supone un viaje al otro, estar dispuesto a abandonar tu zona de confort y mudarte al contexto ajeno del consumidor.

Mientras más empacamos, menos viajamos: solo nos hemos movido sin deslocalizarnos. El antropólogo viajero se relocaliza. Esto implica no reconocer nuestras propias convicciones y prejuicios y desembarcarlos del viaje, con el fin de poder captar el máximo de experiencias vividas y supuestas de nuestro viaje al consumidor.

El profesional de marketing con orientación etnográfica no espera recibir información desde su oficina o laboratorio, sino que viaja en busca de ella siendo él mismo partícipe de la experiencia. En este viaje se construyen observaciones e inmersiones en puntos de venta, espacios públicos u hogares, y se decodifican los códigos culturales de consumo que brinden las claves de acciones de marketing que se integren naturalmente a la cultura. Son travesías innovadoras cuya misión es romper paradigmas de pensamiento, no confirmarlos.

El etnomarketing es instrumentalizado a partir de la observación y la etnografía, considerando el potencial que ofrece corroborar en el contexto real de vida más allá de la información obtenida en simuladores de mercado (como los grupos focales o encuestas)

La observación y la etnografía aportan un aprendizaje experiencial al ejecutivo en la semiconvivencia con el consumidor, que difícilmente se obtendría desde su centro de trabajo, escuela de negocio de acceso restringido y entorno social inmediato. Asimismo, es una herramienta valiosa también para la implementación de programas de clima y cultura organizacional por el carácter inclusivo de su enfoque.

El innecesario divorcio entre empresa y antropología podría estar privando, más allá de oportunidades de empleo a futuros antropólogos, de nuevos ámbitos de estudio o una posibilidad de aproximarse a la realidad de los sujetos contemporáneos movilizados por el consumo. Esta nueva materia en la urbe permite la apertura de puertas para la ubicación 
de la especialidad en el análisis de la contemporaneidad, y ya no la obsesión por el pasado, entendiendo su potencial creativo.

Debido a los retos de comprensión de las reconfiguraciones culturales que generan los procesos económicos y empresariales, la antropología requiere ser convocada de vuelta a los espacios de consumo o de producción modernos para resolver los dilemas que la nueva era global impone: “El posmodernismo forma parte de la coyuntura global en relación con la cual nos es preciso medir los objetivos, las perspectivas y la pertinencia de un nuevo debate antropológico" (Augé 1998: 46). Este debate obliga a instrumentalizar y generar una serie de conocimientos sobre el nuevo ciudadano y sus alteridades, ya no en las fronteras de la civilización, sino en la re-tribalización del público consumidor y de las nuevas generaciones de trabajadores. 


\section{bibliografía}

\section{Arellano, Rolando}

2010

Al medio hay sitio. Lima: Planeta.

Ariely, Dan

2008

Las trampas del deseo. Buenos Aires: Ariel.

\section{Augé, Marc}

2008

Hacia una antropología de los mundos

contemporáneos. Barcelona: Gedisa.

\section{Augé, Marc}

1998

Los no lugares. Barcelona: Gedisa.

\section{Badot, Olivier y Otros}

The Contribution of Ethnology to Research

in Consumer and Shopper Behavior: toward Ethnomarketing. Londres: Recherche et Applications en Marketing, vol. 24, n 1.

\section{Bourdieu, Pierre}

1988

La distinción: criterio y bases sociales del gusto. Madrid: Alfaguara.

\section{Deleuze, Gilles}

2005

1980

Lógica del sentido. Barcelona: Paidós.

Diálogos. Valencia: Pre-textos

\section{Diaz - Albertini, Javier}

2000

Nueva cultura de trabajo en los jóvenes de la clase media limeña. Lima: Universidad de Lima.

\section{Douglas, Mary y Baron}

Isherwood

El mundo de los bienes: hacia una antro-

pología del consumo. México: Grijalbo.

\section{Foucault, Michael}

\section{García Canclini, Néstor}

\section{Geertz, Clifford}

1989

El antropológo como autor. Barcelona: Paidós.

\section{Hevia, Julio}

2002

Lengua y devenires en pugna. En torno a la posmodernidad. Lima: Universidad de Lima.

\section{Huber, Ludwig}

2002

Consumo, cultura e identidad en el mundo globalizado. Lima: IEP.

\section{Hofstede, Geert}

2005

Cultures and Organizations: Software of the Mind. Segunda Edición. New York:

McGraw Hill.

\section{Hofstede, Geert}

1980

Culture's Consequences: International Differences in Work-Related Values. Beverly Hills: Sage Publications.

\section{Páramo, Dagoberto}

Etnomarketing, La dimensión cultural del marketing. Bogotá: Uninorte.

\section{Pazos, Jacinto}

Micronegoscios y Megamercados, Tesis para optar por el grado de Doctorado en Antropología. Lima: UNMSM.

\section{Signorelli, Amalia} Antropología urbana. Barcelona: Universidad Autónoma Metropolitana.

\section{Veblen, Thorstein}

Teoría de la clase ocios. Madrid: Alianza Editorial.

\section{Fecha de recepción: 17/06/15} Fecha de aceptación: 14/10/15 